\title{
CONDITIONS OF COMPATIBILITY FOR THE SOLID-LIQUID INTERFACE
}

\author{
$\mathrm{BY}$ \\ F. BALDONI AND K. R. RAJAGOPAL \\ Department of Mechanical Engineering, University of Pittsburgh, Pittsburgh, PA
}

\begin{abstract}
The seminal theory of singular surfaces propounded by Hadamard and Thomas is examined within the context of the dynamics of a solid-liquid interface. It is shown that most of the hypotheses upon which Clapeyron's equation is based can be weakened and two generalized versions of it are derived: with and without curvature effects. The remaining part of the paper is mainly focused on the interface conditions for the classical Stefan problem. The counterpart of Clapeyron's equation for such a problem will give an explicit expression for the supercooling temperature without recourse to linearization procedures. Furthermore, a decay law for the latent heat of melting is given which shows, in an explicit way, its complex dependence upon the curvature and the normal speed of the interface. Finally, a transport equation for the interface temperature is derived and a qualitative solution of a simplified version of it is given for the particular case in which the jump in the Helmoltz free energies of the bulk phases is a conserved quantity throughout the field.
\end{abstract}

1. Introduction. One of the early definitions of an interfacial system dates back to the pioneering work of Gibbs [1] and defines it as consisting of two (or more) homogeneous bulk phases separated by a singular surface. A singular surface is in turn defined as a surface with no inner structure through which the bulk quantities may suffer finite discontinuities. When material particles that belong to the surface at some time $t^{*}$ remain on the surface at any time $t \geq t^{*}$, the surface is called "material". There are cases, however, in which the motion of the singular surface in the domain and the motion of the particles in the bulk phases are somewhat independent of each other; this may be the case, for instance, for a shock surface in gas dynamics or the advancing solidification front in a liquid-solid phase transformation.

For the latter class of singular surfaces, when curvature effects are neglected, a classical result of thermostatics states that two phases of the same substance can coexist in equilibrium only if they possess the same Gibbs potential $\zeta$ per unit mass where

$$
\zeta=\varepsilon-\theta \eta+\frac{p}{\rho}
$$

Received May 2, 1994.

(C)1997 Brown University 
$\varepsilon$ is the internal energy, $\theta$ is the temperature, $\eta$ is the entropy, $p$ and $\rho$ the pressure and the density respectively. If we consider only two phases, say phase 1 and phase 2 , and if we suppose that on the common surface of contact the Gibbs potential of the two phases is a function of temperature and pressure, the classical result implies that

$$
\zeta_{1}(\theta, p)-\zeta_{2}(\theta, p)=0
$$

Taking the total differential of (1.1), we obtain

$$
d\left(\zeta_{1}(\theta, p)-\zeta_{2}(\theta, p)\right)=\left(\frac{\partial \zeta_{1}}{\partial \theta}-\frac{\partial \zeta_{2}}{\partial \theta}\right) d \theta+\left(\frac{\partial \zeta_{1}}{\partial p}-\frac{\partial \zeta_{2}}{\partial p}\right) d p=0
$$

With the aid of elementary thermodynamical identities, we can rewrite (1.2) as

$$
\left(\eta_{2}-\eta_{1}\right) d \theta=\left(\frac{1}{\rho_{2}}-\frac{1}{\rho_{1}}\right) d p
$$

which is the celebrated Clapeyron's equation. For a solid-liquid system in equilibrium, (1.3) gives, for example, the variation of the melting temperature with the pressure and, in the case of ice, it was experimentally validated by James and William Thompson in 1849. The remarkable agreement between (1.3) and the experimental results boosted, at that time, the aspirations of the still embrionic science of thermostatics and, as Pippart [2] remarks, "... contributed largely to the spirit of confidence which underlay and encouraged the rapid development of the subject."

To the present time, as long as the starting hypotheses are met, (1.3) has never been disproved. The assumptions on the basis of which (1.3) has been derived are, however, rather severe. Not only do curvature effects have to be negligible (in other words, a flat common interface) but the two phases must be in thermal and mechanical equilibrium which implies, for instance, that neither phase can grow or decrease: the interface must then be not only flat but absolutely static.

More than half a century after the original derivation of Clapeyron, in a fundamental memoir, J. Hadamard [3] laid down the basis of the modern theory of singular surfaces. Apart from the inherent beauty of the theory, several fields in which scattered results connected to singular surfaces had appeared were instantaneously unified: the theory of shock waves, vortex sheets, and waves in elastic solids are just some of the branches of rational mechanics that could then be treated within the context of Hadamard's theory. Surprisingly enough, however, the theory of phase transition, Clapeyron's equation representing an important example, was never included nor treated within the context of Hadamard's formalism and this in spite of the fact that, at least from an historical perspective, it was probably one of the earlier settings where the concept of singular surface was introduced.

Whether, and in what cases, the concept of singular surface has a sound physical basis or just represents a mathematical simplification is still a matter of debate and involves issues of a rather complex nature. For a liquid-gas transformation for instance, in which no change in the symmetry properties of the material is associated with the transition, the idea that a thin transitional layer, rather than a sharp interface, separates the two 
phases is now widely accepted: the sharp interface is then interpreted as a macroscopic view of the region while the thin layer is its microscopic counterpart (cf. Mavrovouniotis and Brenner [4]). For solid-liquid transitions, in which a crystalline structure breaks down to a random assembly of molecules, the existence of such a smooth layer seems doubtful. In any case, the idea of a singular surface can be taken either as a starting point toward more refined theories or as a physically reasonable assumption and, in both cases, Hadamard's theory provides a number of invaluable tools for the mathematical treatment of the problem. Central to such a theory is the notion of interfacial condition of compatibility which is directly derived from Hadamard's lemma and which holds for every quantity defined and continuously differentiable in the subdomains of which the singular surface represents the intersection.

It is then our aim to show, on the one hand, that Clapeyron's equation is, in fact, the interfacial condition of compatibility for the Gibbs potential and, on the other hand, that some of the original assumptions of its derivation can be weakened. It is not, in fact, strictly necessary to require the equality of the Gibbs functions across the singular surface as long as their difference is a function of time only or, in particular, a nonzero constant along the interface. We present, in addition, a more general derivation of the equation which allows for (dissipationless) motions in the bulk phases, for heat conduction across and along the interface as well as the presence of curvature and of anisotropic surface tension.

After some preliminary definitions in Sec. 2, we introduce a generalized form of Clapeyron's equation in Sec. 3. In Sec. 4 it is shown that a more natural setting for the analysis of surface effects is given by the classical Stefan problem while the appropriate thermodynamical potential is now given by the Helmoltz free energy $\phi$. When the interface is no longer flat, the influence of the curvature upon the melting temperature (the so-called geometrical supercooling effect) is naturally recovered and an alternative expression for it, without any recourse to linearization procedures, is given.

The results of Sec. 5 are of a qualitative nature. We first provide an estimate for the average curvature of the interface and briefly discuss its relationship with the thermodynamical conditions along it. Section 6 contains a derivation of a decay law for the latent heat of melting, that is, the quantity of heat released or absorbed during the phase transition; for some class of processes this law shows an exponential decay related to, among others quantities, the normal speed of the interface. The last section presents an equation of evolution for the surface temperature in which the dependence upon the normal speed of the interface is made explicit (the kinetic supercooling effect) and which shows, in spite of some necessary restriction, the often intricate way in which all the relevant thermomechanical variables interact during a solid-liquid phase transformation.

2. Preliminaries. Consider a regular surface $\mathcal{S}(t)$ (that is, a surface with a unique normal vector at each point) moving in a domain $\mathcal{D}(t)$ during the interval of time $(0, T)$. At each instant of time, the surface $\mathcal{S}(t)$ divides the domain $\mathcal{D}(t)$ into two subregions $\mathcal{D}^{+}(t)$ and $\mathcal{D}^{-}(t)$ whose common boundary is $\mathcal{S}(t)$ itself, such that

$$
\mathcal{D}(t)=\mathcal{D}^{+}(t) \cup \mathcal{D}^{-}(t), \quad \mathcal{S}(t)=\mathcal{D}^{+}(t) \cap \mathcal{D}^{-}(t)
$$


In the euclidean space $\mathcal{E}$ the surface $\mathcal{S}(t)$ admits the representation

$$
\mathbf{x}=\mathbf{y}(\mathbf{p}, t)
$$

where $\mathbf{p}$ are the parameters of the surface. For a given value of $t \in T,(2.1)$ defines a surface on which $\mathbf{p}$ forms a curvilinear coordinate system. The velocity of the moving surface $\mathcal{S}(t)$ at time $t$ is given by

$$
\mathbf{u}=\frac{\partial \mathbf{y}}{\partial t}
$$

and the speed of $\mathcal{S}(t)$ in the direction of the unit normal $\mathbf{n}$ is

$$
u_{n}=\mathbf{u} \cdot \mathbf{n}
$$

A surface can be (locally) represented by an equation of the form

$$
f(\mathbf{y}, t)=0
$$

with $\mathbf{y} \in \mathcal{S}(t)$. In terms of this representation

$$
\mathbf{n}=\frac{\nabla f}{\|\nabla f\|}, \quad u_{n}=-\frac{1}{\|\nabla f\|} \frac{\partial f}{\partial t} .
$$

We shall assume, from now on, that the singular surface $\mathcal{S}(t)$ is smooth enough to ensure the global validity of the representation given by (2.2). If $\psi$ is a function defined and continuous in $\mathcal{D}^{ \pm}(t)$ but discontinuous across the surface, we define

$$
\psi^{+}=\lim _{\mathbf{x} \rightarrow \mathbf{y}^{+}} \psi(\mathbf{x}, t), \quad \psi^{-}=\lim _{\mathbf{x} \rightarrow \mathbf{y}^{-}} \psi(\mathbf{x}, t),
$$

where the limits are taken along an arbitrary path from the interior of $\mathcal{D}^{+}(t)$ (or $\mathcal{D}^{-}(t)$ ) to $\mathcal{S}(t)$. We then define the discontinuity of $\psi$ across the surface as

$$
[\psi](\mathbf{y}, t)=\psi^{+}-\psi^{-}
$$

and the average as

$$
\langle\psi\rangle(\mathbf{y}, t)=\frac{\psi^{+}+\psi^{-}}{2} .
$$

The basic result in the theory of singular surfaces is due to Hadamard [3] which states that if the function $\psi$ is continuously differentiable in $\mathcal{D}^{+}(t)$ and $\mathcal{D}^{-}(t)$, then also $\psi^{+}$ and $\psi^{-}$are differentiable on $\mathcal{S}(t)$. We state the main lemma and refer the reader to Truesdell and Toupin [5] or to the classical work of Hadamard [3] for the proof.

LEMMA. Let $\mathcal{S}(t)$ be a singular surface for $\psi(\mathbf{x}, t)$ and let $\mathbf{z}=\mathbf{Z}(s, t)$ be a smooth curve on $\mathcal{S}(t)$ with curvilinear coordinate $s$. Then

$$
\begin{aligned}
& \frac{\partial \psi^{+}}{\partial s}=\left(\frac{\partial \psi}{\partial \mathbf{x}}\right)^{+} \frac{\partial \mathbf{z}}{\partial s} \\
& \frac{\partial \psi^{-}}{\partial s}=\left(\frac{\partial \psi}{\partial \mathbf{x}}\right)^{-} \frac{\partial \mathbf{z}}{\partial s}
\end{aligned}
$$

where $\mathbf{x}$ is the spatial coordinate system. 
Taking the difference of (2.7.1) and (2.7.2) we obtain

$$
\frac{\partial[\psi]}{\partial s}=\left[\frac{\partial \psi}{\partial \mathbf{x}}\right] \frac{\partial \mathbf{z}}{\partial s}
$$

while, by taking the sum and dividing by two

$$
\frac{\partial\langle\psi\rangle}{\partial s}=\left\langle\frac{\partial \psi}{\partial \mathbf{x}}\right\rangle \frac{\partial \mathbf{z}}{\partial s} .
$$

Equation (2.8), which is known as Hadamard's lemma, can be rewritten as

$$
\operatorname{grad}[\psi] \cdot \mathbf{t}=[\operatorname{grad} \psi] \cdot \mathbf{t}
$$

where $\mathbf{t}(s, t)$ is the tangent vector to the curve $\mathbf{z}$ and since it is valid for every $t$, it implies that

$$
[\operatorname{grad} \psi]=\operatorname{grad}[\psi]+\left[\frac{\partial \psi}{\partial n}\right] \mathbf{n}
$$

where

$$
\frac{\partial \psi}{\partial n}=\frac{\partial \psi}{\partial \mathbf{x}} \cdot \mathbf{n}
$$

In particular, if $\psi$ is continuous across $\mathcal{S}(t)$, then it follows from $(2.10)$ that

$$
[\operatorname{grad} \psi]=\left[\frac{\partial \psi}{\partial n}\right] \mathbf{n}
$$

which is better known as Maxwell's theorem [5]. We now introduce the displacement derivative (cf. Thomas [6]) given by

$$
\frac{\delta \psi}{\delta t}=\frac{\partial \psi}{\partial t}+u_{n} \frac{\partial \psi}{\partial n}
$$

The meaning of the displacement derivative is simple: given a function defined on the singular surface, it gives the rate of change of the function as perceived by an observer moving with the normal velocity of the surface itself. If we apply $(2.12)$ to $\psi^{+}$and $\psi^{-}$ separately and subtract the result, we obtain

$$
\frac{\delta[\psi]}{\delta t}=\left[\frac{\partial \psi}{\partial t}\right]+u_{n}\left[\frac{\partial \psi}{\partial n}\right] .
$$

Clearly, a similar expression holds for the average. On the basis of a classification which, to our knowledge, was first introduced by Thomas [6], Hadamard's lemma and the displacement derivative are, respectively, the geometrical and kinematical conditions of compatibility for the singular surface $\mathcal{S}(t)$. It is, however, necessary to take into account the equations of balance and the constitutive equations for the material under consideration in order to obtain the dynamical conditions whose validity, contrary to the previous ones, holds only locally; hence, they will assure the possibility of propagation of a surface of discontinuity only "in the small". 
3. The generalized Clapeyron's equation. Consider a system composed of a liquid and a solid phase which, under suitable boundary conditions, is undergoing a phase transformation process (from liquid to solid for example). The balance of mass, linear momentum, and energy at the liquid-solid interface $\mathcal{S}(t)$ are given by (cf. Truesdell and Toupin [5])

$$
\begin{gathered}
{\left[\rho\left(v_{n}-u_{n}\right)\right]=0} \\
{\left[\rho\left(v_{n}-u_{n}\right) \mathbf{v}\right]-[\mathbf{T n}]+\operatorname{div}_{\mathcal{S}} \mathbf{T}_{\mathcal{S}}=\mathbf{0}} \\
{\left[\rho\left(v_{n}-u_{n}\right)\left(\varepsilon+\frac{|\mathbf{v}|^{2}}{2}\right)\right]+[\mathbf{q}-\mathbf{T} \mathbf{v}] \cdot \mathbf{n}=0}
\end{gathered}
$$

where $\mathbf{v}=v_{t} \mathbf{t}+v_{n} \mathbf{n}$ is the velocity of a particle on the singular surface, $\mathbf{T}=-p \mathbf{1}+\mathbf{T}^{e}$ is the bulk stress tensor, $\mathbf{q}$ is the heat flux vector, $\operatorname{div}_{\mathcal{S}}$ is the surface divergence operator, and $\mathbf{T}_{\mathcal{S}}$ is the surface stress tensor that we will assume to be given by

$$
\mathbf{T}_{\mathcal{S}}=\sigma \mathbf{1}
$$

where $\sigma$ is the surface tension. ${ }^{1}$ If we define the quantity

$$
\Gamma=\rho^{-}\left(v_{n}^{-}-u_{n}\right)=\rho^{+}\left(v_{n}^{+}-u_{n}\right),
$$

it follows from (3.2) and (3.3) that

$$
\begin{gathered}
\Gamma\left[v_{n}\right]-[\mathbf{T n}] \cdot \mathbf{n}=-2 \sigma \aleph, \\
\Gamma\left[v_{t}\right]-\left[\mathbf{T}^{e} \mathbf{n}\right] \cdot \mathbf{t}=-\mathbf{t} \cdot \nabla \sigma, \\
\Gamma\left[\varepsilon+\frac{|\mathbf{v}|^{2}}{2}\right]+[\mathbf{q}-\mathbf{T} \mathbf{v}] \cdot \mathbf{n}=0,
\end{gathered}
$$

where $\aleph$ is the mean curvature of the surface. To this set of equations we add the jump statement of the second law which we shall assume to be in the form of the ClausiusDuhem inequality which reads

$$
\Gamma[\eta]+\left[\frac{\mathbf{q} \cdot \mathbf{n}}{\theta}\right] \leq 0
$$

where the temperature $\theta$, from now on, will be assumed continuous across the singular surface $\mathcal{S}(t)$. Let us now consider the case in which

$$
\mathbf{T}=-p \mathbf{1}
$$

\footnotetext{
${ }^{1}$ It should be noticed that, with the necessary exception of the surface tension $\sigma$, no additional properties of the interface have been introduced. The interface is modeled essentially as a singular surface in that, at an instant, a particle on the singular surface moves with a velocity different from that of the singular surface. However, in those cases in which the interface $\mathcal{S}(t)$ is taken to represent a material surface, interfacial properties like the surface density $\rho_{\mathcal{S}}$, surface entropy $\eta_{\mathcal{S}}$, etc., would have to be posited (cf. Kosinski [7]).
} 
in the bulk phases while on the solid-liquid interface we will assume constant surface tension. In this case, from (3.4), (3.5), and (3.6) we obtain

$$
\begin{gathered}
{[p]=-2 \sigma \aleph-\Gamma\left[v_{n}\right],} \\
{\left[v_{t}\right]=0,} \\
\Gamma\left[\varepsilon+\frac{v_{n}^{2}}{2}\right]+[\mathbf{q} \cdot \mathbf{n}]+\left[p v_{n}\right]=0 .
\end{gathered}
$$

Moreover, since by virtue of (3.4)

$$
\Gamma\left[\frac{p}{\rho}\right]=\left[p\left(v_{n}-u_{n}\right)\right]
$$

it follows from (3.8) and (3.10) that

$$
\Gamma\left[\chi+\frac{v_{n}^{2}}{2}-v_{n} u_{n}\right]+[\mathbf{q} \cdot \mathbf{n}]-2 \sigma \aleph u_{n}=0
$$

where $\chi=\varepsilon+p / \rho$ is the enthalpy. Due to the continuity of $u_{n}$ across the interface $\mathcal{S}(t)$, the following identity holds:

$$
\left[\frac{v_{n}^{2}}{2}-v_{n} u_{n}\right]=\left[\frac{\left(v_{n}-u_{n}\right)^{2}}{2}\right]
$$

so that, if we now subtract (3.7) from (3.12) we find

$$
\Gamma\left[\zeta+\frac{\left(v_{n}-u_{n}\right)^{2}}{2}\right]-2 \sigma \aleph u_{n} \geq 0
$$

where $\zeta$ is the Gibbs function. Let us now restrict our attention to the case of a perfectly flat interface. We notice from (3.4) that

$$
\Gamma\left[v_{n}\right]=\left[\rho\left(v_{n}-u_{n}\right)^{2}\right]
$$

so that (3.8) and (3.13) become

$$
\begin{aligned}
{\left[p+\rho\left(v_{n}-u_{n}\right)^{2}\right] } & =0, \\
\Gamma\left[\zeta+\frac{\left(v_{n}-u_{n}\right)^{2}}{2}\right] & \geq 0 .
\end{aligned}
$$

Let

$$
\begin{aligned}
& P=p+\rho\left(v_{n}-u_{n}\right)^{2}, \\
& E=\varepsilon-\frac{\left(v_{n}-u_{n}\right)^{2}}{2} .
\end{aligned}
$$


From (3.14) then follows

$$
\begin{gathered}
{[P]=0,} \\
\Gamma[\Upsilon] \geq 0
\end{gathered}
$$

where

$$
\Upsilon=E+\frac{P}{\rho}-\theta \eta=\zeta+\frac{U^{2}}{2}
$$

and

$$
U=u_{n}-v_{n}
$$

is the speed of the interface relative to a fixed reference system. It is important to notice that, in spite of our restriction on the stress tensor, the conditions of classical thermostatics do not obtain which would require both thermal and mechanical equilibrium in the bulk phases though the values of variables such as pressure and density are allowed to be different from one phase to another. In our case, since it is evident from (3.15) that no such equilibrium has been postulated and, as a matter of fact, it could not be: heat is conducted from the liquid to the solid phase and both phases are in motion. It is our aim, however, to examine the behaviour of the solidifying system in the case in which

$$
[\Upsilon]=g(t) \neq 0
$$

that is, when the jump in the (modified) Gibbs free energy of the two phases, which still satisfies the inequality (3.15), is a function of time only. Toward this end, we apply Hadamard's lemma (2.8) to the function $\Upsilon$ which, in the bulk regions and on the basis of the previous definitions, we will assume to be a function of $\theta(\mathbf{x}, t), P(\mathbf{x}, t)$, and $U^{2}$. We then have

$$
\frac{\partial[\Upsilon]}{\partial s}=\left\{\left[\frac{\partial \Upsilon}{\partial \theta} \frac{\partial \theta}{\partial \mathbf{x}}\right]+\left[\frac{\partial \Upsilon}{\partial P} \frac{\partial P}{\partial \mathbf{x}}\right]+\left[\frac{\partial \Upsilon}{\partial\left(U^{2}\right)} \frac{\partial\left(U^{2}\right)}{\partial \mathbf{x}}\right]\right\} \frac{\partial \mathbf{z}}{\partial s} .
$$

By virtue of (3.17) the term on the left-hand side of (3.18) is zero. It is easy to show moreover that, from the definition of jump and average of a function $f$, the following identity holds:

$$
\left[f_{1} f_{2}\right]=\left[f_{1}\right]\left\langle f_{2}\right\rangle+\left\langle f_{1}\right\rangle\left[f_{2}\right] .
$$

Using (3.19), we can rewrite (3.18) as

$$
\begin{gathered}
\left\{\left[\frac{\partial \Upsilon}{\partial \theta}\right]\left\langle\frac{\partial \theta}{\partial \mathbf{x}}\right\rangle+\left\langle\frac{\partial \Upsilon}{\partial \theta}\right\rangle\left[\frac{\partial \theta}{\partial \mathbf{x}}\right]+\left[\frac{\partial \Upsilon}{\partial P}\right]\left\langle\frac{\partial P}{\partial \mathbf{x}}\right\rangle\right\} \frac{\partial \mathbf{z}}{\partial s} \\
+\left\{\left\langle\frac{\partial \Upsilon}{\partial P}\right\rangle\left[\frac{\partial P}{\partial \mathbf{x}}\right]+\left[\frac{\partial \Upsilon}{\partial\left(U^{2}\right)} \frac{\partial\left(U^{2}\right)}{\partial \mathbf{x}}\right]\right\} \frac{\partial \mathbf{z}}{\partial s}=0
\end{gathered}
$$

and apply Hadamard's lemma and (2.9) to the variables $\theta$ and $P$ : since they are continuous across the interface, the second and fourth term in (3.20) will vanish such that

$$
\left\{\left[\frac{\partial \Upsilon}{\partial \theta}\right]\left\langle\frac{\partial \theta}{\partial \mathbf{x}}\right\rangle+\left[\frac{\partial \Upsilon}{\partial P}\right]\left\langle\frac{\partial P}{\partial \mathbf{x}}\right\rangle+\left[\frac{\partial \Upsilon}{\partial\left(U^{2}\right)} \frac{\partial\left(U^{2}\right)}{\partial \mathbf{x}}\right]\right\} \frac{\partial \mathbf{z}}{\partial s}=0
$$


However, from (3.16) we have

$$
\frac{\partial \Upsilon}{\partial \theta}=\frac{\partial \zeta}{\partial \theta}, \quad \frac{\partial \Upsilon}{\partial p}=\frac{\partial \zeta}{\partial p}
$$

and

$$
\frac{\partial \Upsilon}{\partial p}=\frac{\partial \Upsilon}{\partial P} \frac{\partial}{\partial p}\left\{p+\rho(\theta, p) U^{2}\right\}=\frac{\partial \Upsilon}{\partial P}\left(1+\frac{\partial \rho}{\partial p} U^{2}\right)
$$

so that (3.21) becomes

$$
\left\{\left[\frac{\partial \zeta}{\partial \theta}\right]\left\langle\frac{\partial \theta}{\partial \mathbf{x}}\right\rangle+\left[\frac{\partial \zeta}{\partial p}\left(1+\frac{\partial \rho}{\partial p} U^{2}\right)^{-1}\right]\left\langle\frac{\partial P}{\partial \mathbf{x}}\right\rangle+\left[U \frac{\partial U}{\partial \mathbf{x}}\right]\right\} \frac{\partial \mathbf{z}}{\partial s}=0 .
$$

From the continuity of $\theta$ and $P$ across $\mathcal{S}(t)$ it also follows that

$$
\begin{gathered}
\langle\theta\rangle=\theta^{ \pm}=\vartheta(s, t), \\
\langle P\rangle=P^{ \pm}=\mathcal{P}(s, t) .
\end{gathered}
$$

Hence, applying (2.9) to $\theta$ and $P$ and by virtue of standard thermodynamic identities for the function $\zeta(\theta, p),(3.22)$ reduces to

$$
[\eta] \frac{\partial \vartheta}{\partial s}=\left[\frac{1}{\rho\left(1+\rho K_{T} U^{2}\right)}\right] \frac{\partial \mathcal{P}}{\partial s}+\frac{1}{2} \frac{\partial\left[U^{2}\right]}{\partial s}
$$

where

$$
K_{T}=\frac{1}{\rho} \frac{\partial \rho(\theta, p)}{\partial p}
$$

is the coefficient of isothermal compressibility. Clearly, in the limiting case of a static interface $\left(u_{n}=0\right)$ and in the absence of motion in the bulk phases $\left(v_{n}=0\right)$ we have $P=p($ and $\mathcal{P}=\wp)$ and $(3.24)$ becomes

$$
\left[\frac{1}{\rho}\right] \frac{\partial \wp(s, t)}{\partial s}=[\eta] \frac{\partial \vartheta(s, t)}{\partial s} .
$$

Equation (3.24) (or (3.26)) is the desired generalization of the classical Clapeyron's equation (cf. Guggenheim [8]) but, despite the formal resemblance to its famous counterpart from thermostatics, it is quite different from it. Equation (3.26) holds in the presence of nonuniform fields of pressure, temperature, density, and velocity while Clapeyron's equation is only valid under the condition of strict equilibrium (uniformity of every field). As such, (3.26) provides the variation of the interface temperature as a function of the corresponding variation of pressure and interface speed along the singular surface and shows that even in the presence of a constant pressure (along the interface) the surface temperature can be varied by increasing (or decreasing) the velocity at which the process of solidification is taking place.

When $\aleph \neq 0$ and the surface tension is not constant the previous arguments can be modified as follows. Without loss of generality, we will restrict our attention to the case 
$\Gamma \geq 0$. The condition given by (3.17) will still hold but the function $g(t)$ is now subject to the constraint

$$
\Gamma g(t) \geq \sup _{\mathbf{x} \in \mathcal{S}(t)}\left|2 \sigma \aleph u_{n}\right|
$$

The function $E$ and generalized pressure $P$ are now given by

$$
\begin{gathered}
E=\varepsilon-\frac{\left|\mathbf{v}-u_{n} \mathbf{n}\right|^{2}}{2}=\varepsilon-\frac{|\mathbf{V}|^{2}}{2}, \\
P=p+\rho\left|\mathbf{v}-u_{n} \mathbf{n}\right|^{2}=p+\rho|\mathbf{V}|^{2} .
\end{gathered}
$$

Clearly, when $\sigma$ is not constant, Eq. (3.9) does not hold anymore but instead we have

$$
\Gamma\left[v_{t}\right]=-\mathbf{t} \cdot \nabla \sigma
$$

In this case it is a simple matter to show that (3.24) is replaced by

$$
[\eta] \frac{\partial \vartheta}{\partial s}=\left[\frac{1}{\rho\left(1+\rho K_{T}|\mathbf{V}|^{2}\right)}\right] \frac{\partial\langle P\rangle}{\partial s}+\frac{1}{2} \frac{\partial\left[|\mathbf{V}|^{2}\right]}{\partial s}+\left\langle\frac{1}{\rho\left(1+\rho K_{T}|\mathbf{V}|^{2}\right)}\right\rangle \frac{\partial}{\partial s}\left(2 \sigma \aleph-\left[\rho v_{t}^{2}\right]\right) .
$$

For an anisotropic crystal the surface tension can vary with the surface temperature and with the orientation of the crystal planes. Hence, since a given smooth surface can be imbedded in a smooth family of surfaces, $f=$ constant, we let

$$
\sigma=\sigma(\mathbf{n}, \vartheta)
$$

In the absence of the motion in the bulk phases, from (3.27) and the previous assumption we obtain

$$
[\eta] \frac{\partial \vartheta}{\partial s}=\left[\frac{1}{\rho}\right] \frac{\partial\langle P\rangle}{\partial s}+2\left\langle\frac{1}{\rho}\right\rangle\left\{\sigma \frac{\partial \aleph}{\partial s}+\aleph\left(\frac{\partial \sigma}{\partial \mathbf{n}} \cdot \frac{\partial \mathbf{n}}{\partial s}+\frac{\partial \sigma}{\partial \vartheta} \frac{\partial \vartheta}{\partial s}\right)\right\}
$$

In the two-dimensional case the singular surface reduces to a plane curve, and from the first Frenet formula [9],

$$
\frac{\partial \mathbf{n}}{\partial s}=-\aleph \mathbf{t}
$$

and from (3.28) we find

$$
\left([\eta]-2\left\langle\frac{1}{\rho}\right\rangle \aleph \frac{\partial \sigma}{\partial \vartheta}\right) \frac{\partial \vartheta}{\partial s}=\left[\frac{1}{\rho}\right] \frac{\partial\langle P\rangle}{\partial s}+2\left\langle\frac{1}{\rho}\right\rangle\left(\sigma \frac{\partial \aleph}{\partial s}-\aleph^{2} \mathbf{t} \cdot \frac{\partial \sigma}{\partial \mathbf{n}}\right) .
$$

4. The generalized Clapeyron's equation for the Stefan problem. The generality of Eq. (3.27) is, to some extent, illusory. Certainly Eq. (3.27), which incorporates curvature and anisotropy effects, already gives some information about the supercooling effect, namely the variation of the freezing temperature with the curvature of the interface. If, however, the singular surface has a nonzero curvature, as is apparent from (3.16), a modified Gibbs free energy $\Upsilon$ which is independent of $s$ is much less likely to appear: if, in fact, the curvature is not constant, from (3.8) the jump in the pressure will depend 
upon the curvilinear coordinate $s$ and, from (3.16), the jump in the modified Gibbs free energy as well. An additional difficulty arises from the fact that, in the presence of a nonzero curvature, the pressure field is no longer continuous across the interface and, as apparent from (3.28), the variable $\mathcal{P}$ is replaced by the average $\langle P\rangle$ which, in general, will depend upon the curvature of the interface. Thus, if we want to include curvature effects into the basic physical framework and explicitly determine their influence, it is necessary to modify the basic assumptions that lead to (3.15) and this can only be done by allowing some degree of continuity in a dependent variable other than pressure.

An ideal setting which provides most of the previous requirements is given by the Stefan model which postulates, as its basis, a negligible motion in the bulk phases. In such a case $(3.1),(3.4),(3.5),(3.6)$, and (3.7) reduce to

$$
\begin{gathered}
{[\rho] u_{n}=0,} \\
{[p]=-2 \sigma \aleph,} \\
\sigma=\text { constant, } \\
-\rho^{ \pm} u_{n}[\varepsilon]+[\mathbf{q} \cdot \mathbf{n}]=0, \\
-\rho^{ \pm} u_{n}[\eta]+\left[\frac{\mathbf{q} \cdot \mathbf{n}}{\theta}\right] \leq 0 .
\end{gathered}
$$

Since the normal velocity of the interface $u_{n}$ is generally different from zero, (4.1) tells us that the density is continuous across the interface while, if we subtract (4.4) from (4.5) we obtain

$$
\rho u_{n}[\phi] \leq 0
$$

where $\phi=\varepsilon-\theta \eta$ is the Helmholtz free energy. It may then be of some interest to explore, following the guidelines of the previous section, the case in which the jump in the Helmoltz free energy is a nonzero function of time along the (now curved) interface $\mathcal{S}(t)$.

We apply again Hadamard's lemma (2.8) to the function $\phi$ which we will now suppose to be a function of the temperature $\theta(\mathbf{x}, t)$ and of the density $\rho(\mathbf{x}, t)$. We have

$$
\left\{\left[\frac{\partial \phi}{\partial \theta} \frac{\partial \theta}{\partial \mathbf{x}}\right]+\left[\frac{\partial \phi}{\partial \rho} \frac{\partial \rho}{\partial \mathbf{x}}\right]\right\} \frac{\partial \mathbf{z}}{\partial s}=0 .
$$

Since both the temperature and the density are continuous across the interface, using the same arguments as before and from (2.9) applied to $\theta$ and $\rho$ we find

$$
\left[\frac{\partial \phi}{\partial \theta}\right] \frac{\partial \vartheta(s, t)}{\partial s}+\left[\frac{\partial \phi}{\partial \rho}\right] \frac{\partial \rho(s, t)}{\partial s}=0
$$

But

$$
\frac{\partial \phi(\theta, \rho)}{\partial \theta}=-\eta, \quad \frac{\partial \phi(\theta, \rho)}{\partial \rho}=\frac{p}{\rho^{2}},
$$

so that, from (4.8) and (4.2) we obtain

$$
\frac{\partial \vartheta(s, t)}{\partial s}=-\frac{2 \sigma \aleph}{\rho^{2}[\eta]} \frac{\partial \rho(s, t)}{\partial s} .
$$


Equation (4.9) can be viewed as the counterpart, for the Stefan problem, of the generalized Clapeyron's equation (3.24). Let us now apply Hadamard's lemma to the pressure $p(\theta, \rho):$ this gives

$$
\frac{\partial[p]}{\partial s}=\left[\frac{\partial p}{\partial \theta}\right] \frac{\partial \vartheta}{\partial s}+\left[\frac{\partial p}{\partial \rho}\right] \frac{\partial \rho}{\partial s} .
$$

It is now easy to prove that

$$
\frac{\partial p}{\partial \theta}=\frac{\rho \Delta c}{\theta \beta}
$$

where $\beta$ is the coefficient of thermal expansion and $\Delta c=c_{p}-c_{v}$ is the difference between the specific heats at constant pressure and density, and by virtue of (4.2), Eq. (4.10) can be rewritten as

$$
\frac{\rho}{\vartheta}\left[\frac{\Delta c}{\beta}\right] \frac{\partial \vartheta}{\partial s}+\frac{1}{\rho}\left[\frac{1}{K_{T}}\right] \frac{\partial \rho}{\partial s}=-2 \sigma \frac{\partial \aleph}{\partial s} .
$$

If we now denote with $D$ the determinant of the coefficients of the system given by (4.9) and (4.12), that is,

$$
D(\vartheta, \aleph, s, t)=\frac{[\eta]}{2 \sigma}\left[\frac{1}{K_{T}}\right] \vartheta-\aleph\left[\frac{\Delta c}{\beta}\right],
$$

we need to consider two different cases.

If there exists an interval $\mathcal{C}^{*} \subset \mathcal{C}$ where the determinant $D$ vanishes, from (4.13), we obtain the interface temperature as

$$
\vartheta=\frac{2 \sigma \aleph}{[\eta]}\left[\frac{\Delta c}{\beta}\right]\left[\frac{1}{K_{T}}\right]^{-1} \quad \text { in } \mathcal{C}^{*}
$$

while, from (4.12) and (4.9) we conclude that

$$
\frac{\partial \aleph}{\partial s}=0 \quad \text { in } \mathcal{C}^{*}
$$

that is, $\aleph=f(t)$ in the interval of interest. If we then substitute (4.14) into (4.9) and take (4.3) and the previous results into account we find

$$
\frac{\partial}{\partial s}\left(\frac{1}{\rho}\right)=[\eta] \frac{\partial}{\partial s}\left(\frac{1}{[\eta]}\left[\frac{\Delta c}{\beta}\right]\left[\frac{1}{K_{T}}\right]^{-1}\right) \quad \text { in } \mathcal{C}^{*}
$$

In the case in which $D(\vartheta, \aleph, s, t) \neq 0$, from (4.9) and (4.12) we obtain

$$
\frac{\partial \vartheta}{\partial s}=\frac{2 \sigma \vartheta \aleph}{D(\vartheta, \aleph, s, t) \rho} \frac{\partial \aleph}{\partial s}
$$

and

$$
\frac{\partial \rho}{\partial s}=-\frac{\rho[\eta] \vartheta}{D(\vartheta, \aleph, s, t) \rho} \frac{\partial \aleph}{\partial s}
$$

Every coefficient in (4.15) and (4.16) is expected to depend upon the curvilinear coordinates $s$ and upon the time $t$; hence, unless this dependence is made explicit, the above nonlinear system can, in general, be solved only by numerical means. However, some 
indications about the possible behaviour of the solutions, can be obtained by examining a particular case in which all the thermomechanical variables appearing in (4.15) and (4.16) are independent of $t$ with the additional constraint that

$$
\left[\frac{\Delta c}{\beta}\right]=0
$$

Equations (4.15) and (4.16) then reduce to

$$
\frac{1}{\rho} \frac{d \rho}{d s}=-\frac{1}{2 \sigma\left[1 / K_{T}\right]} \frac{d \aleph}{d s}
$$

and

$$
\frac{d \vartheta}{d s}=\frac{[\eta]}{\left[1 / K_{T}\right]} \frac{\aleph}{\rho} \frac{d \aleph}{d s}
$$

Hence, from (4.17) we obtain

$$
\rho(s)=\rho\left(s_{0}\right) \exp \left\{-\frac{1}{2 \sigma} \int_{s_{0}}^{s} \frac{1}{\left[1 / K_{T}\right]} \frac{\partial \aleph}{\partial q} d q\right\}
$$

while (4.18) gives

$$
\vartheta(s)=\vartheta\left(s_{0}\right)+\frac{1}{\rho\left(s_{0}\right)} \int_{s_{0}}^{s} \frac{[\eta]}{\left[1 / K_{T}\right]} \exp \left(\frac{1}{2 \sigma} \int_{s_{0}}^{u} \frac{1}{\left[1 / K_{T}\right]} \frac{\partial \aleph}{\partial q} d q\right) \aleph \frac{\partial \aleph}{\partial u} d u
$$

From (4.20) the interdependence between curvature and melting temperature becomes explicit and it shows a somewhat unexpected feature: the average of the limiting values of the density itself depends upon the geometry (curvature) of the interface. Equation (4.20) moreover places no restriction upon the order of magnitude of the interfacial curvature and differs from the linearizations usually adopted in the phase-transformations literature.

5. An estimate for the average curvature. Consider the identity

$$
\Delta[\phi]=[\phi](s, t)-[\phi]\left(s_{0}, t\right)=\int_{s_{0}}^{s} \frac{\partial[\phi]}{\partial s} d s
$$

with $s \geq s_{0}$. It follows from (5.1) and from the Schwartz inequality that

$$
(\Delta[\phi])^{2} \leq\left(\int_{s_{0}}^{s}\left(\frac{\partial[\phi]}{\partial s}\right)^{2} d s\right)\left(s-s_{0}\right) .
$$

For the function $\phi(\theta, \rho)$, Hadamard's lemma (2.8) reads

$$
\frac{\partial[\phi]}{\partial s}=\left[\frac{\partial \phi}{\partial \theta}\right] \frac{\partial \vartheta}{\partial s}+\left[\frac{\partial \phi}{\partial \rho}\right] \frac{\partial \rho}{\partial s} .
$$


By introducing the specific volume $\nu=\rho^{-1}$ we can rewrite (5.3) as

$$
\frac{\partial[\phi]}{\partial s}=-[\eta] \frac{\partial \vartheta}{\partial s}+[p] \frac{\partial \nu}{\partial s}
$$

We then substitute (5.4) into (5.2) and apply the Minkowski inequality to obtain

$$
(\Delta[\phi])^{2} \leq\left(s-s_{0}\right)\left\{\left(\int_{s_{0}}^{s}[\eta]^{2}\left(\frac{\partial \vartheta}{\partial s}\right)^{2} d s\right)^{1 / 2}+2 \sigma\left(\int_{s_{0}}^{s} \aleph^{2}\left(\frac{\partial \nu}{\partial s}\right)^{2} d s\right)^{1 / 2}\right\}
$$

where (4.2) has been employed. It then follows from Hölder's inequality that

$$
\begin{aligned}
\frac{(\Delta[\phi])^{2}}{\left(s-s_{0}\right)} \leq & \left(\int_{s_{0}}^{s}[\eta]^{4} d s\right)^{1 / 4}\left(\int_{s_{0}}^{s}\left(\frac{\partial \vartheta}{\partial s}\right)^{4} d s\right)^{1 / 4} \\
& +2 \sigma\left(\int_{s_{0}}^{s} \aleph^{4} d s\right)^{1 / 4}\left(\int_{s_{0}}^{s}\left(\frac{\partial \nu}{\partial s}\right)^{4} d s\right)^{1 / 4}
\end{aligned}
$$

If the thermal conductivity $k$ is constant across the singular surface $\mathcal{S}(t)$ we also have

$$
k \frac{\partial \vartheta}{\partial s}=k\langle\nabla \theta\rangle \cdot \mathbf{t}=\langle k \nabla \theta\rangle \cdot \mathbf{t}=-\langle\mathbf{q}\rangle \cdot \mathbf{t}
$$

and similarly

$$
\frac{\partial \rho}{\partial s}=\langle\nabla \rho\rangle \cdot \mathbf{t}
$$

such that, if we introduce the norm

$$
\|\cdot\|=\left(\int_{s_{0}}^{s}(\cdot)^{4} d s\right)^{1 / 4}
$$

we can rewrite (5.6) as

$$
2 \sigma\|\aleph\| \geq \frac{1}{\|\langle\nabla \rho\rangle \cdot \mathbf{t}\|}\left\{\frac{(\Delta[\phi])^{2}}{\left(s-s_{0}\right)}-\|[\eta]\| \frac{\|\langle\mathbf{q}\rangle \cdot \mathbf{t}\|}{k}\right\} .
$$

The main conclusion we can draw from (5.8) is then the following: If equality were to hold in (5.8), for a given (fixed) temperature and density field in the bulk phases, the average curvature of the interface increases with the variation of the jump in the Helmholtz free energy along the singular surface. Since, moreover, the condition

$$
[\phi](s, t)=0
$$

represents, for the Stefan problem, the condition of equilibrium between phases, any departure from such a condition can be assumed as an approximate measure of the degree of disequilibrium of the bulk phases. 
6. Spatial decay of the latent heat of melting. The energy equation for the Stefan problem is given by the classical heat conduction equation, i.e.,

$$
\rho c_{v} \frac{\partial \theta}{\partial t}=k \Delta \theta
$$

If we assume that the specific heat at constant volume $c_{v}$ and the thermal conductivity $k$ are continuous across the singular surface $\mathcal{S}(t)$, by taking the jump of $(6.1)$ across the surface we obtain

$$
\rho c_{v}\left[\frac{\partial \theta}{\partial t}\right]=k[\Delta \theta]
$$

Since the temperature $\theta$ is continuous across $\mathcal{S}(t)$,

$$
\frac{\delta[\theta]}{\delta t}=0
$$

so that, by definition of the displacement derivative,

$$
\left[\frac{\partial \theta}{\partial t}\right]=-u_{n}\left[\frac{\partial \theta}{\partial n}\right]
$$

It can then be shown that (cf. Chadwick and Powdrill [10])

$$
[\Delta \theta]=-2 \aleph\left[\frac{\partial \theta}{\partial n}\right]+\left[\frac{\partial^{2} \theta}{\partial n^{2}}\right]
$$

Hence, from (6.2), (6.3), and (6.4) it follows that

$$
\left(2 \aleph-\frac{\rho c_{v}}{k} u_{n}\right)\left[\frac{\partial \theta}{\partial n}\right]=\left[\frac{\partial^{2} \theta}{\partial n^{2}}\right]
$$

However, using the identity (cf. Chadwick and Powdrill [10])

$$
\left[\frac{\partial^{2} \theta}{\partial t^{2}}\right]=-2 u_{n} \frac{\delta}{\delta t}\left[\frac{\partial \theta}{\partial n}\right]-\frac{\delta u_{n}}{\delta t}\left[\frac{\partial \theta}{\partial n}\right]+u_{n}^{2}\left[\frac{\partial^{2} \theta}{\partial n^{2}}\right],
$$

equation (6.5) can be rewritten as

$$
2 \frac{\delta \mathcal{T}}{\delta t}+\left(\frac{\rho c_{v}}{k} u_{n}^{2}-2 \aleph u_{n}+\frac{1}{u_{n}} \frac{\delta u_{n}}{\delta t}\right) \mathcal{T}+\frac{\mathcal{T}}{u_{n}}=\frac{\mathcal{Q}}{u_{n}}
$$

where

$$
\left[\frac{\partial \theta}{\partial n}\right]=\mathcal{T}, \quad\left[\frac{\partial^{2} \theta}{\partial t^{2}}\right]=\mathcal{Q} .
$$

We now want to consider the case in which

$$
\frac{\partial \mathcal{T}}{\partial t}=0, \quad u_{n}=\text { constant }
$$


It follows that

$$
\frac{\delta \mathcal{T}}{\delta t}=u_{n} \frac{\partial \mathcal{T}}{\partial n}
$$

We recall that if we define the metric tensor $\mathbf{g}$ on $\mathcal{S}(t)$ whose $\mathbf{p}$-components are

$$
g^{\alpha \beta}=y_{i, \alpha} y_{i, \beta}
$$

the displacement derivative of the normal vector $\mathbf{n}$ is given by (cf. Truesdell and Toupin $[5])$

$$
\frac{\delta \mathbf{n}}{\delta t}=-g^{\alpha \beta} \mathbf{y}_{, \alpha} u_{n, \beta} .
$$

If $u_{n, \beta}=0$, that is, if $u_{n}$ is constant on the surface $\mathcal{S}(t)$, then (6.7) and the definition of the displacement derivative imply that the normal vector $\mathbf{n}$ is constant along the normal trajectory, which means that the normal trajectories are straight lines. This in turn implies that the family of surfaces $\{\mathcal{S}(t)\}_{t \in T}$ will form a family of parallel surfaces. A typical example of a similar situation is given by the polyhedral shapes where, in the course of growth, the shape of the crystal is always similar to the original one. Let us now suppose that at $t=t_{0}$ the mean and Gaussian curvatures of the wavefront $\Sigma\left(t_{0}\right)$ are given by $\aleph_{0}(s)$ and $\omega_{0}(s)$ respectively. Then, if the normal distance $n$ is measured from $\Sigma\left(t_{0}\right)$, the mean curvature of $\mathcal{S}(t)$ is given by (cf. Thomas [11])

$$
\aleph(n, s)=\frac{\aleph_{0}(s)-n \omega_{0}(s)}{1-2 n \aleph_{0}(s)+n^{2} \omega_{0}(s)} .
$$

By virtue of the previous results (6.6) becomes

$$
2 \frac{\partial \mathcal{T}}{\partial n}+\left(\frac{\rho c_{v}}{k} u_{n}-2 \frac{\aleph_{0}-n \omega_{0}}{1-2 n \aleph_{0}+n^{2} \omega_{0}} u_{n}\right) \mathcal{T}+\frac{\mathcal{Q}}{u_{n}}=0
$$

where the dependence of the various coefficients in (6.9) upon $s$ is understood. Since $n\left(t_{0}\right)=n_{0}=0$, we integrate $(6.9)$ to obtain

$$
\mathcal{T}(n, s)=\Lambda(n, s)-\frac{\mathcal{T}_{0}(s)}{\left(1-2 n \aleph_{0}+n^{2} \omega_{0}\right)^{u_{n} / 2}} \exp \left(-\frac{u_{n}}{2} \int_{0}^{n} \frac{\rho c_{v}}{k}(n, s) d n\right)
$$

where $\Lambda(n, s)$ is a particular integral of (6.9). Finally, from (4.2) and (4.4) we have

$$
\rho u_{n}[\chi]=-2 \sigma \aleph u_{n}+[\mathbf{q} \cdot \mathbf{n}]=-2 \sigma \aleph u_{n}-k \mathcal{T}
$$

and, from (6.10)

$$
[\chi](n, s)=-\frac{k \Lambda(n, s)}{\rho u_{n}}-\frac{2 \sigma \aleph}{\rho}-\frac{\rho[\chi]_{0}+2 \sigma \aleph_{0}}{\rho\left(1-2 n \aleph_{0}+n^{2} \omega_{0}\right)^{u_{n} / 2}} \exp \left(-\frac{u_{n}}{2} \int_{0}^{n} \frac{\rho c_{v}}{k}(n, s) d n\right)
$$

which gives the decay law for the latent heat of melting $[\chi]$. The particular integral $\Lambda(n, s)$ will dominate the behaviour of the solution for large $n$ while, in a neighborhood of $n=0$, the second and third term in (6.11) are the most significant. In such a range and for a positive interface speed $u_{n}$, since the argument under the integral sign in (6.11) is never negative, the latent heat decreases (at least) exponentially with the interface speed $u_{n}$ and with the norm of the kernel $\rho c_{v} / k$. 
7. An equation of evolution for the interface temperature. Consider

$$
\frac{\delta[\phi]}{\delta t}=\left[\frac{\partial \phi}{\partial t}\right]+u_{n}\left[\frac{\partial \phi}{\partial n}\right]
$$

and assume that, within the framework of the Stefan problem given by (4.1)-(4.5), the Helmholtz free energy $\phi$ in the bulk phases is a function of temperature and density; then, by arguments similar to those used in Sec. 3, we can rewrite (7.1) as

$$
\frac{\delta[\phi]}{\delta t}=-[\eta] \frac{\delta\langle\theta\rangle}{\delta t}+\left[\frac{2 \sigma \aleph}{\rho^{2}}\right] \frac{\delta\langle\rho\rangle}{\delta t}
$$

or, by using the property of continuity of $\theta$ and $\rho$ across the interface, as

$$
\frac{\delta[\phi]}{\delta t}=-[\eta] \frac{\delta \vartheta}{\delta t}+\frac{2 \sigma \aleph}{\rho^{2}} \frac{\delta \rho}{\delta t}
$$

where $\langle\theta\rangle=\vartheta$ and $\langle\rho\rangle=\rho$. From Hadamard's lemma however, we also have

$$
\frac{\partial[\phi]}{\partial s}=-[\eta] \frac{\partial \vartheta}{\partial s}+\frac{2 \sigma \aleph}{\rho^{2}} \frac{\partial \rho}{\partial s}
$$

If we now cross differentiate (7.2) and (7.3) and subtract the final result we find

$$
\frac{2 \sigma}{\rho^{2}} \frac{\partial \aleph}{\partial s} \frac{\delta \rho}{\delta t}-\frac{2 \sigma}{\rho^{2}} \frac{\partial \rho}{\partial s} \frac{\delta \aleph}{\delta t}-\frac{\partial[\eta]}{\partial s} \frac{\delta \vartheta}{\delta t}+\frac{\delta[\eta]}{\delta t} \frac{\partial \vartheta}{\partial s}=0
$$

Notice that in the absence of curvature, (7.4) admits the solution (cf. Courant and Hilbert [12])

$$
\vartheta=\vartheta([\eta])
$$

It follows from (7.2), (7.3), and (7.4) that

$$
\frac{\partial \mathcal{K}}{\partial s} \frac{\delta \vartheta}{\delta t}-\frac{\delta \mathcal{K}}{\delta t} \frac{\partial \vartheta}{\partial s}=\frac{\Pi}{\aleph[\eta]}
$$

where

$$
\begin{aligned}
& \Pi=\frac{\partial \aleph}{\partial s} \frac{\delta[\phi]}{\delta t}-\frac{\delta \aleph}{\delta t} \frac{\partial[\phi]}{\partial s}, \\
& \mathcal{K}=\ln \frac{\aleph}{[\eta]} .
\end{aligned}
$$

If we introduce the velocity $V_{c}$ defined as

$$
V_{c}=\left(\frac{\delta \mathcal{K}}{\delta t}\right) /\left(\frac{\partial \mathcal{K}}{\partial s}\right)
$$

from (7.5) we obtain

$$
\frac{\partial \vartheta}{\partial t}+u_{n} \frac{\partial \vartheta}{\partial n}-V_{c} \frac{\partial \vartheta}{\partial s}=\frac{\Pi}{\aleph[\eta]}\left(\frac{\partial \mathcal{K}}{\partial s}\right)^{-1}=\Pi^{*}
$$


Equation (7.6), whose form strongly resembles a transport equation, can be solved for the unknown interface temperature $\vartheta$ only if the functional form of $[\phi]$ is known. To this end, consider the case in which the quantity $[\phi]$ is conserved, that is,

$$
\frac{d}{d t} \int_{S(t)}[\phi] d S=0
$$

for every $S(t)$. Since, as we already remarked, the jump in the Helmholtz free energy can be taken to represent the driving force of the interfacial motion (recall that the condition $[\phi]=0$ gives an interface in equilibrium) the case given by (7.8) may describe an interface steadily driven throughout the field or, in other words, two bulk phases which, in spite of their relative growth, preserve the same difference in the Helmoltz free energy with each other.

From the transport theorem for singular surfaces, suitably simplified due to the zero velocity of the particles on the singular surface (cf. Kosinski [7]), we have

$$
\int_{S(t)}\left(\frac{\delta[\phi]}{\delta t}-2 \aleph u_{n}[\phi]\right) d S=0
$$

or, since the surface $S(t)$ is arbitrary

$$
\frac{\delta[\phi]}{\delta t}-2 \aleph u_{n}[\phi]=0
$$

Notice that (7.9) admits the equilibrium solution $[\phi]=0$.

Let the surface $S(t)$ be given by $\mathrm{x}=\phi(s, t)$. Then, for a function $f(s, t)$, we have

$$
\frac{\partial f(s, t)}{\partial t}=\frac{\partial \bar{f}[\phi(s, t), t]}{\partial t}+u_{n} \frac{\partial \bar{f}[\phi(s, t), t]}{\partial n}=\frac{\delta \bar{f}[\phi(s, t), t]}{\delta t}
$$

where

$$
\frac{\partial}{\partial n}(\cdot)=\mathbf{n} \cdot \frac{\partial}{\partial \phi}(\cdot)
$$

We set $[\phi]=\Phi(s, t)$ and obtain the solution of (7.9) in the form

$$
\Phi(s, t)=\Phi(s, 0) e^{2 \int_{0}^{t} \aleph u_{n} d t^{\prime}} .
$$

On setting $\theta=\bar{\theta}(s, t)$ we can rewrite (7.7) as

$$
\frac{\partial \bar{\theta}}{\partial t}-V_{c}(s, t) \frac{\partial \bar{\theta}}{\partial s}=\pi^{*}(s, t)
$$

For $t$ close to zero, we shall approximate (7.11) by

$$
\frac{\partial \bar{\theta}}{\partial t}-V_{c}(s, 0) \frac{\partial \bar{\theta}}{\partial s}=\pi^{*}(s, 0)
$$

Now, suppose that

$$
\bar{\theta}=\theta_{1}(s, t)+\theta_{2}(s) .
$$


On substituting (7.13) into (7.12), we obtain

$$
\frac{\partial \bar{\theta}_{1}}{\partial t}=V_{c}(s, \theta) \frac{\partial \bar{\theta}_{1}}{\partial s}-V_{c}(s, 0) \frac{\partial \bar{\theta}_{2}}{\partial s}=\pi^{*}(s, 0) .
$$

We can ensure (7.14) provided

$$
\begin{gathered}
\frac{\partial \theta_{1}}{\partial t}-\bar{V}_{c}(s, 0) \frac{\partial \theta_{1}}{\partial s}=0, \\
\bar{V}_{c}(s, 0) \frac{d \theta_{2}}{d s}=-\pi^{*}(s, 0) .
\end{gathered}
$$

Thus, for $\bar{V}_{c}(s, 0) \neq 0$, the solution of $(7.12)$ will be given by

$$
\bar{\theta}(s, t)=\Theta\left(t+\int \frac{d s^{\prime}}{V_{c}\left(s^{\prime}, 0\right)}\right)-\int \frac{\pi^{*}\left(s^{\prime}, 0\right)}{V_{c}\left(s^{\prime}, 0\right)} d s^{\prime}
$$

where $\Theta$ is an arbitrary function of the argument.

It follows from (7.6.2), (7.9), (6.8), and (7.13) that

$$
\Pi^{*}(0,0, s)=-u_{n}\left\{\omega_{0} \frac{\partial[\phi]_{0}}{\partial s}+\frac{\partial}{\partial s}\left([\phi]_{0} \aleph_{0}^{2}\right)\right\}\left\{[\eta]_{0} \frac{\partial \aleph_{0}}{\partial s}-\aleph_{0}\left(\frac{\partial[\eta]}{\partial s}\right)_{0}\right\}^{-1}
$$

The two terms that appear in (7.18) contain the basic quantities upon which the interface temperature $\vartheta$ is expected to depend: the curvature $\aleph$, the entropy of melting $[\eta]$, and the interface speed $u_{n}$. The first term, which we may call the kinematical contribution, takes into account the variation of the surface temperature due to the motion of the surface, and its wave-like form shows that the surface temperature propagates along a characteristic.

The second term, or the geometrical contribution, depends exclusively upon the surface properties and on the normal speed $u_{n}$. Equation (7.18) provides an expression for the dependence of the interface temperature upon $s$ under conditions away from equilibrium and shows, moreover, that the kinetic supercooling can be included in the formulation of the Stefan problem within the framework of continuum thermomechanics.

\section{REFERENCES}

[1] J. W. Gibbs, Scientific Papers, Dover, New York, 1961

[2] A. B. Pippard, Elements of Classical Thermodynamics, Cambridge University Press, Cambridge, 1966

[3] J. Hadamard, Leçons sur la propagation des ondes et les équations de l'hydrodynamique, Hermann, Paris, 1903

[4] G. M. Mavrovouniotis and H. Brenner, A Micromechanical Investigation of Interfacial Transport Processes. I. Interfacial Conservation Equation, Philos. Trans. Royal Soc. (345) 1675, 165-207 (1993)

[5] C. Truesdell and R. Toupin, The Classical Field Theories, Handbuch der Physik, vol. 3/III, Springer-Verlag, Berlin, 1964

[6] T. Y. Thomas, Plastic flow and fracture in solids, Academic Press, New York, 1961

[7] W. Kosinski, Field singularities and wave analysis in continuum mechanics, Ellis-Horwood Limited, London, 1986 
[8] E. A. Guggenheim, Thermodynamics: an advanced treatment for chemists and physicists, NorthHolland, Amsterdam, 1986

[9] J. J. Stoker, Differential Geometry, Wiley-Interscience, New York, 1969

[10] P. Chadwick and B. Powdrill, Singular surfaces in linear thermoelasticity, Internat. J. Engrg. Sci. 3, 561-595 (1965)

[11] T. Y. Thomas, Concepts from Tensor Analysis and Differential Geometry, Academic Press, New York, 1961

[12] R. Courant and D. Hilbert, Methods of Mathematical Physics, Vol. II, Wiley-Interscience, New York, 1962 